\title{
An Influence of the Parameters of Pre-Stressing and Winkler Elastic Foundation on a Stability of the Geometrically Nonlinear Column under Specific Load
}

\author{
J. SZMidla* AND I. CieŚlińSKA-GĄSIOR \\ Częstochowa University of Technology, al. Armii Krajowej 19, 42-201 Częstochowa, Poland \\ Doi: 10.12693/APhysPolA.138.170 \\ *e-mail: cieslinskailona@wp.pl
}

\begin{abstract}
The paper presents the results of theoretical and numerical studies on the issue of stability of a geometrically nonlinear column subjected to follower force directed towards the positive pole (specific load). Due to the geometric nonlinearity in the model, a recti- and curvilinear form of static equilibrium can be distinguished. The loss of rectilinear form is local and does not lead to complete destruction of the column, but significantly reduces its maximum load. The paper proposes to include in the model the initial pre-stressing of the system and the local Winkler elastic foundation in order to increase the value of bifurcation load. The boundary problem was formulated using the Hamilton principle and variation calculus properties, and then solved using the perturbative method.
\end{abstract}

topics: loss of rectilinear form, pre-stressing, Winkler elastic foundation, static criterion of stability

\section{Introduction}

In the field of stability studies of geometrically non-linear slender systems, various cases of conservative loading were considered: Euler's [1], specific [2] and non-conservative load including Beck's generalized load [3]. In Ref. [4], the system was subjected to a load subjected to a follower force directed to the positive pole - the specific load case [5]. The influence of the Winkler elastic foundation and initial pre-stressing has been widely described in the scientific literature. Various types of supporting bases were considered in the tests: along the entire length of all system bars (total) [6], the entire length of selected system bars (partial) [7] or on a certain section of bars along the length of the system (local) [7, 8]. In Ref. [9], the combined effect of rotational and translational elasticity on the critical load value of the column under the follower load was considered. In article [10], the frequencies vibrations were examined and the maximum deflections of the geometrically nonlinear Timoshenko beam resting completely on the Winkler-Pasternak elastic foundation were determined.

Local and global loss of rectilinear form of static equilibrium of a geometrically nonlinear system, discussed in [11] is the result of a comparative analysis of the bifurcation load value of this system and the critical force of the comparative column (geometrically linear system), with the assumed flexural stiffness asymmetry.

\section{Physical model}

Figure 1 presents the physical model of a geometrically nonlinear column resting locally on a Winkler elastic foundation.

The column (Fig. 1) consists of two external rods with lengths $l_{1}, l_{2}$ and a central rod symmetrically placed relative to the two external rods. In order to model the local support on an elastic foundation of elasticity $K$, the inner rod is divided into three parts with $l_{3}, l_{4}, l_{5}$, where

$$
l_{1}=l_{2}=l_{3}+l_{4}+l_{5} \text {. }
$$

Taking into account the presented description, the system under consideration consists of five members with the distribution of flexural and compressive stiffness

$$
\begin{aligned}
& (E J)_{1}=(E J)_{2}, \quad(E A)_{1}=(E A)_{2}, \\
& (\rho A)_{1}=(\rho A)_{2}, \quad(E J)_{3}=(E J)_{4}=(E J)_{5}, \\
& (E A)_{3}=(E A)_{4}=(E A)_{5}, \\
& (\rho A)_{3}=(\rho A)_{4}=(\rho A) .
\end{aligned}
$$

The paper assumes a lower bending stiffness $(E J)_{3}$ of the internal bar with respect to the external bars: $(E J)_{3} \leq(E J)_{1}+(E J)_{2}$. The follower force directed to the positive pole is carried out by the loading and receiving heads of the circular outline. The direction of the external load $P$ passes through a point $O$ lying on the undeformed axis of the 


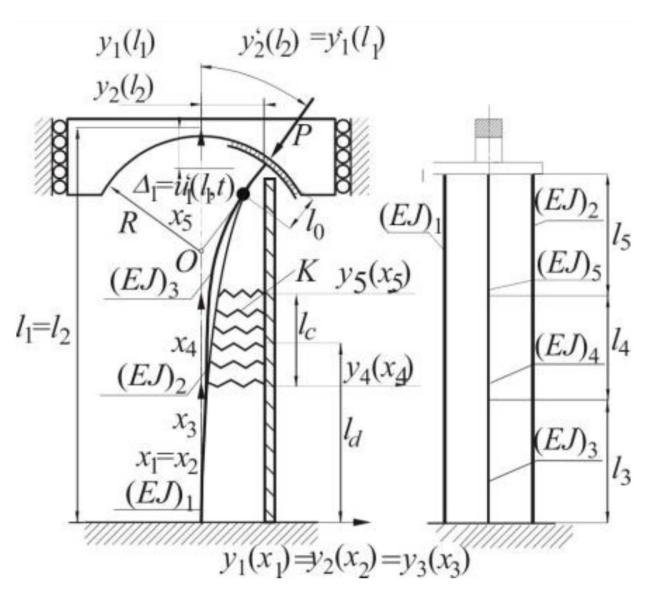

Fig. 1. Physical model of pre-stressed geometrically nonlinear column with local Winkler elastic foundation support $\mathrm{NW}_{\mathrm{M}} \mathrm{SP}$.

column and is tangent to the deflection line of the free end, where the column is connected to the load receiving head via an element of length $l_{0}$. In order to model the elastic base, parameters describing its location and size have been introduced

$$
l_{c}=l_{4}, l_{d}=l_{3}+\frac{l_{4}}{2} .
$$

The asymmetry of flexural stiffness of the system is described by first Eq. (6), it was assumed that the total flexural stiffness of the system is a constant (second Eq. (6)):

$$
\mu=\frac{(E J)_{3}}{(E J)_{1}+(E J)_{2}}, \quad \sum_{k=1}^{3}(E J)_{3}=\text { idem. }
$$

\section{Mathematical model}

The total potential energy $V$ of the system consists of energy of: internal forces, bending elasticity, elastic base rigidity and external load components

$$
\begin{aligned}
V & =\frac{1}{2} \sum_{i=1}^{5}(E J)_{i} \int_{0}^{l_{i}}\left[y_{i}^{\mathrm{II}}\left(x_{i}\right)\right]^{2} \mathrm{~d} x_{i} \\
& +\frac{1}{2} \sum_{i=1}^{5}(E A)_{i} \int_{0}^{l_{i}}\left[\frac{1}{2}\left(y_{i}^{I}\left(x_{i}\right)\right)^{2}+u_{i}^{I}\left(x_{i}\right)\right]^{2} \mathrm{~d} x_{i} \\
& -P u_{1}\left(l_{1}, t\right)+\frac{1}{2} P\left(R-l_{0}\right)\left[y_{1}^{\mathrm{I}}\left(l_{1}\right)\right]^{2} \\
& +\frac{1}{2} K \int_{0}^{l_{4}}\left(y_{4}\left(x_{4}\right)\right)^{2} \mathrm{~d} x_{4}
\end{aligned}
$$

where $y_{i}^{I}\left(x_{i}\right), u_{i}\left(x_{i}\right)$ are lateral and longitudinal displacements. The transverse displacement equations are

$$
\begin{aligned}
& (E J)_{j} y_{j}^{\mathrm{IV}}\left(x_{j}\right)+S_{j} y_{j}^{\mathrm{II}}\left(x_{j}\right)=0, \quad j=1,2,3,5 \\
& (E J)_{4} y_{4}^{\mathrm{IV}}\left(x_{4}\right)+S_{4} y_{4}^{\mathrm{II}}\left(x_{4}\right)+K y_{4}\left(x_{4}\right)=0
\end{aligned}
$$

where $S_{i}$ - internal force of $i$-th member of the system.

By definition, the longitudinal forces in the outer bars and individual parts of the middle bar are:

$$
\begin{aligned}
& S_{i}(t)=-(E A)_{i}\left[u_{i}^{\mathrm{I}}\left(x_{i}\right)+\frac{1}{2}\left(y_{i}^{\mathrm{I}}\left(x_{i}\right)\right)^{2}\right], \\
& i=1 \ldots 5 .
\end{aligned}
$$

Differential equations of motion towards the nondeformed axis of the column are written in the form

$$
\frac{\partial}{\partial x_{i}}\left[u_{i}^{\mathrm{I}}\left(x_{i}\right)+\frac{1}{2}\left(y_{i}^{\mathrm{I}}\left(x_{i}\right)\right)^{2}\right]=0, \quad i=1 \ldots 5 .
$$

Double integration of Eqs. (11) in their respective ranges and taking into account Eq. (10) enabled the determination of formula describing longitudinal displacements of individual bars of the system

$$
u_{i}\left(x_{i}\right)-u_{i}(0)=-\frac{S_{i}}{(E A)_{i}} x_{i}-\frac{1}{2} \int_{0}^{x_{i}}\left(y_{i}^{\mathrm{I}}\left(x_{i}\right)\right)^{2} \mathrm{~d} x_{i} .
$$

Geometric and natural boundary conditions of the considered system (Fig. 1):

$$
\begin{aligned}
& y_{3}\left(l_{3}\right)=y_{4}(0), \quad u_{3}\left(l_{3}\right)=u_{4}(0) \text {, } \\
& y_{4}\left(l_{4}\right)=y_{5}(0), \quad u_{4}\left(l_{4}\right)=u_{5}(0) \text {, } \\
& y_{3}^{\mathrm{I}}\left(l_{3}\right)=y_{4}^{\mathrm{I}}(0), \quad y_{4}^{\mathrm{I}}\left(l_{4}\right)=y_{5}^{\mathrm{I}}(0), \\
& y_{1}\left(l_{1}\right)=y_{2}\left(l_{2}\right)=y_{5}\left(l_{5}\right) \text {, } \\
& u_{1}\left(l_{1}\right)=u_{2}\left(l_{2}\right)=u_{5}\left(l_{5}\right), \\
& y_{1}^{\mathrm{I}}(0)=y_{2}^{\mathrm{I}}(0)=y_{5}^{\mathrm{I}}(0)=0, \\
& y_{1}^{\mathrm{I}}\left(l_{1}\right)=y_{2}^{\mathrm{I}}\left(l_{2}\right)=y_{5}^{\mathrm{I}}\left(l_{5}\right) \text {, } \\
& y_{1}(0)=y_{2}(0)=y_{3}(0)=0 \text {, } \\
& u_{1}(0)=u_{2}(0)=u_{3}(0)=0, \\
& y_{3}^{\mathrm{II}}\left(l_{3}\right)=y_{4}^{\mathrm{II}}(0), \quad y_{3}^{\mathrm{III}}\left(l_{3}\right)=y_{4}^{\mathrm{III}}(0) \text {, } \\
& y_{4}^{\mathrm{II}}\left(l_{4}\right)=y_{5}^{\mathrm{II}}(0), \quad y_{4}^{\mathrm{III}}\left(l_{4}\right)=y_{5}^{\mathrm{III}}(0) \text {, } \\
& (E J)_{1} y_{1}^{\mathrm{III}}\left(l_{1}\right)+(E J)_{2} y_{2}^{\mathrm{III}}\left(l_{2}\right)+(E J)_{5} y_{5}^{\mathrm{III}}\left(l_{5}\right) \\
& -\frac{1}{R-l_{0}}\left[(E J)_{1} y_{1}^{\mathrm{II}}\left(l_{1}\right)+(E J)_{2} y_{2}^{\mathrm{II}}\left(l_{2}\right)\right. \\
& \left.+(E J)_{5} y_{5}^{\mathrm{II}}\left(l_{5}\right)\right]=0 \text {, } \\
& y_{1}\left(l_{1}\right)=\left(R-l_{0}\right) y_{1}^{I}\left(l_{1}\right), \sum_{k=1}^{3} S_{k}-P=0 \\
& S_{1}=S_{2}=P \frac{(E A)_{1}}{\sum_{k=1}^{3}(E A)_{k}} \pm S_{0}, \\
& S_{3}=S_{4}=S_{5}=S-S_{1} .
\end{aligned}
$$

The distribution of internal forces in the bars of the system is described by formula (14). 


\section{Results of numerical computations}

The following column designations are introduced taking into account geometric and physical parameters, whereby:

- $\mathrm{NW}_{\mathrm{M}} \mathrm{SP}\left(\mu, R^{*}, K^{*}, l_{c}^{*}, l_{d}^{*}, S_{0}^{*}\right.$ - a geometrically nonlinear column resting locally on a Winkler elastic foundation of a stiffness coefficient $K^{*}$, subjected to pre-stressing $S_{0}^{*}$ and external load $P$,

- NP $\left(\mu, R^{*}\right)$ — geometrically nonlinear column - comparative system, without pre-stressing and elastic foundation,

- L2P $\left(\mu, R^{*}\right)$ - geometrically linear column comparative system, without an internal bar (Fig. 1),

- dimensionless parameter of: bifurcation load $\lambda_{b}^{*}$, critical load $\lambda_{c}^{*}$ of $\mathrm{NW}_{\mathrm{M}} \mathrm{SP}$ system and linear L2P geometric systems respectively, elastic foundation parameter $K^{*}$, parameter of the loading head $R^{*}$, parameter of initial compression $S_{0}^{*}$ refer to the total bending stiffness and length of the system

$$
\begin{aligned}
\lambda_{b}^{*} & =\frac{P_{b} l_{1}^{2}}{\sum_{k=1}^{3}(E J)_{k}}, \quad \lambda_{c}^{*}=\frac{P_{k r} l_{1}^{2}}{\left.\sum_{k=1}^{3}(E J)\right)_{k}}, \\
K^{*} & =\frac{K l_{1}^{4}}{\sum_{k=1}^{3}(E J)_{k}}, \quad R^{*}=\frac{R-l_{0}}{l_{1}}, \\
S_{0}^{*} & =\frac{S_{0} l_{1}^{2}}{\sum_{k=1}^{3}(E J)_{k}} .
\end{aligned}
$$

Figure 2 shows the range of changes in bifurcation load of NP, NW $\mathrm{MSP}$ systems and critical load of the L2P column in function of the coefficient of flexural stiffness asymmetry $\mu$. When $\lambda_{b}^{*}<\lambda_{c}^{*}$, that means $\mu<\mu_{G R}, \mu \in\left(0, \mu_{G R}\right)$, there is a phenomenon of local loss of rectilinear form of static equilibrium [6]. The effect of the initial pre-stressing and the elastic base resulted in an increase in the value of the dimensionless parameter of the bifurcation load of $\mathrm{NW}_{\mathrm{M}} \mathrm{SP}$ columns above the critical load of the L2P system. The increase in bifurcation load results in a lowering of the coefficient $\mu_{G R}$ value, which leads to a reduction in the area of local loss of rectilinear static equilibrium. Pre-stressing in the area of global loss of rectilinear static equilibrium is not recommended if the condition $\lambda_{b_{N P}}^{*}>\lambda_{b_{N W_{M} S P}}^{*}$ is met.

Figure 3 presents the bifurcation load (line 1) of the $\mathrm{NW}_{\mathrm{M}} \mathrm{SP}$ system as a function of pre-stressing (parameter $S_{0}^{*}$ ) with the assumed flexural stiffness asymmetry in the scope of local loss of rectilinear form of static equilibrium. Curves $(3,4)$ additionally

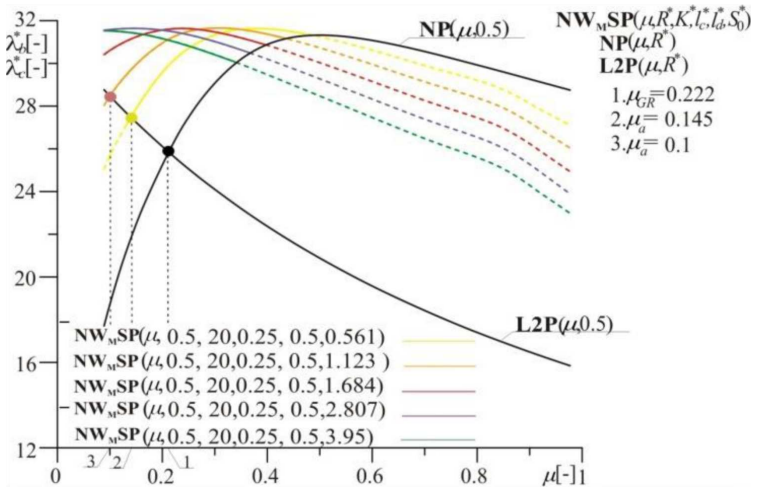

Fig. 2. Bifurcation load parameter of the prestressed $\mathrm{NW}_{\mathrm{M}} \mathrm{SP}$ column as a function of the flexural stiffness asymmetry when $K^{*}=60$.

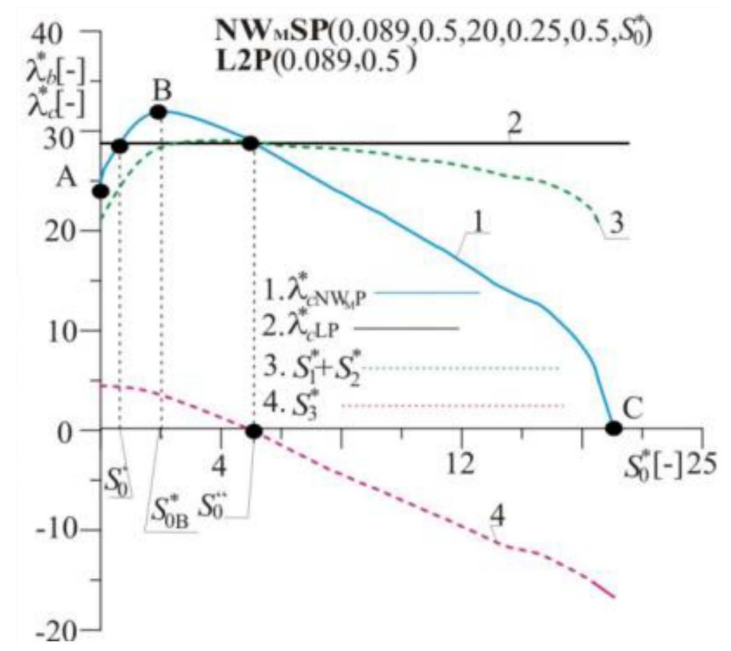

Fig. 3. The parameter of bifurcation load $\lambda_{b}^{*}$ of the $\mathrm{NW}_{\mathrm{M}} \mathrm{SP}$ system and critical load $\lambda_{c}^{*}$ as a function of internal force $S_{0}^{*}$.

determined the range of changes in internal forces $S_{1}^{*}+S_{2}^{*}$ of the external bars of the system and the internal bar $S_{3}^{*}$. The critical load value $\lambda_{c}^{*}$ of the linear (comparative) system is determined by line 2 . It has been shown that in the pre-stressing range $S_{0}^{*} \in\left(S_{0}^{\prime}, S_{0}^{\prime \prime}\right)$, the bifurcation load of the column under consideration has been increased above the critical force of the linear system (line 2). Under the condition $S_{0}^{*}>S_{0}^{\prime \prime}$, the loss of a rectilinear form of static equilibrium is the result of a buckling of a pair of rods characterized by greater bending stiffness $\left.(E J)_{1}+(E J)_{2}\right)$. In the case of $S_{0}^{*} \in\left(0, S_{0}^{\prime \prime}\right)$, the loss of the rectilinear form of static equilibrium is determined by the combined effect of the initial stressing, external load and the elastic foundation of the system, which is associated with the assumed asymmetry of flexural stiffness between bars of the geometrically nonlinear system and the elasticity of the elastic base. Based on the tests carried out, it has been shown that pre-compression should be carried out in the range of $S_{0}^{*} \in\left(S_{0}^{\prime}, S_{0}^{\prime \prime}\right)$. 


\section{Conclusions}

After carrying out numerical calculations, it was found that the considered system is characterized by local or global loss of rectilinear form of static equilibrium. The parameter of flexural stiffness asymmetry $\mu$ affects the value of bifurcation force of a considered column, the smaller value of the flexural stiffness asymmetry, the smaller force that the column carries. The ranges of pre-stressing and elastic base parameters at which an increase in the bifurcation load of the column above the limit of local loss of rectilinear form of static equilibrium is obtained, was determined. It was found that prestressing of the column in the whole possible range is not recommended from the point of view of the obtained bifurcation force. This applies especially to large $S_{0}^{*}$ values, for which the results are opposite to the expected ones (significant reduction of the critical load). Pre-stressing should be used for columns characterized by local loss of rectilinear static equilibrium. Appropriate selection of prestressing parameters and the Winkler elastic foundation increases the area of global loss of stability.

\section{References}

[1] J. Przybylski, L. Tomski, Vibration of an Initially Prestressed Compound Column under Axial Compression, Vol. 9, Elsevier, 1992, p. 263.

[2] L. Tomski, J. Szmidla, J. Theor. Appl. Mech. 44, 279 (2006).
[3] J.A. Hernández-Urrea, J.D. AristizábalOchoa, J. Sound Vibrat. 312, 789 (2008).

[4] J. Szmidla, Drgania Swobodne $i$ Stateczność Układów Smuktych Poddanych Obciażeniu Swoistemu, Monografie 165, Wydawnictwo Politechnika Częstochowska, Częstochowa 2009 (in Polish).

[5] R. Bogacz, Sz. Imiełowski, L. Tomski, Dynamics of Continua - Int. Symp., Physikzentrum Bad Honnef, 1996, p. 45.

[6] J.D. Aristizabal-Ochoa, in: Proc. 16th ASCE Engineering Mechanics Conf., Seattle (WA), 2003, p. 783.

[7] D. Thambiratnam, Y. Zhuge, Comp. Struct. 60, 971 (1996).

[8] I. Elishakoff, X. Wang, Appl. Mech. Rev. 58, 117 (2005).

[9] Z. Celep, J. Appl. Mech. 47, 116 (1980).

[10] V. Stojanović, Commun. Nonlinear Sci. Num. Simulat. 28, 66 (2015).

[11] J. Szmidla, I. Cieślińska-Gasior, Modelowanie Inżynierskie 28, 69 (2016). 